\title{
circular RNAs 0000515 and 0011385 as potential biomarkers for disease monitoring and determining prognosis in pancreatic ductal adenocarcinoma
}

\author{
HANQING WU ${ }^{1}$, BO WANG ${ }^{2}$, LI WANG $^{1}$ and YINKAI XUE ${ }^{1}$ \\ Departments of ${ }^{1}$ Emergency Surgery and ${ }^{2}$ Pancreatic Surgery, Union Hospital, Tongji Medical College, \\ Huazhong University of Science and Technology, Wuhan, Hubei 430022, P.R. China
}

Received November 19, 2020; Accepted September 13, 2021

DOI: $10.3892 / \mathrm{ol} .2021 .13174$

\begin{abstract}
Pancreatic ductal adenocarcinoma (PDAC) is extremely fatal and potential biomarkers for precision medicine of patients with PDAC are yet to be elucidated. Moreover, the clinical values of circular RNAs (circRNAs) in PDAC management are yet to be investigated. The aim of the present study was to perform a secondary analysis of two PDAC public datasets (GSE69362 and GSE79634), to identify the candidate circRNAs, to validate the expression of these circRNAs, and to determine their association with the clinicopathological characteristics and survival of patients with PDAC. A total of 60 patients with PDAC were retrospectively reviewed in the present study. The expression levels of these candidate circRNAs were detected in PDAC tissues and paired adjacent normal tissues via reverse transcription-quantitative PCR analysis. In addition, the clinicopathological characteristics and overall survival (OS) of patients with PDAC were recorded. Bioinformatics analysis identified 22 overlapping differentially expressed (DE) circRNAs between the GSE69362 and GSE79634 datasets, among which nine DEcircRNAs with accordant expression trends (the DEcircRNAs that were upregulated or downregulated in tumor tissues compared with paired adjacent normal tissues in both datasets) were selected as candidate circRNAs, including
\end{abstract}

Correspondence to: Dr Yinkai Xue, Department of Emergency Surgery, Union Hospital, Tongji Medical College, Huazhong University of Science and Technology, 1277 Jiefang Avenue, Wuhan, Hubei 430022, P.R. China

E-mail: zaliang405740@163.com

Abbreviations: PDAC, pancreatic ductal adenocarcinoma; circRNA, circular RNA; DEcircRNAs, differentially expressed circRNAs; GO, Gene Ontology; KEGG, Kyoto Encyclopedia of Genes and Genomes; LNM, lymph node metastasis; OS, overall survival; ROC, receiver operating characteristic; BFAR, bifunctional apoptosis regulator

Key words: PDAC, circRNA, expression profiles, clinicopathological characteristic, survival circ_0000515, circ_0000517, circ_0000520, circ_0000514, circ_0011385, circ_0055033, circ_0072088,circ_0003528 and circ_0008514. In the 60 patients with PDAC, the expression levels of circ_0000515, circ_0000517, circ_0000520, circ_0000514, circ_0011385, circ_0055033, circ_0072088 and circ_0003528 were notably upregulated in PDAC tissues compared with paired adjacent normal tissues. Furthermore, circ_0000515, circ_0000520, circ_0000514, circ_0011385 and circ_0072088 were positively associated with T stage, $\mathrm{N}$ stage and/or TNM stage in patients with PDAC. Notably, circ_0000515 and circ_0011385 were negatively associated with OS in patients with PDAC. Taken together, the results of the present study suggest that circ_0000515 and circ_0011385 may serve as prognostic biomarkers for patients with PDAC.

\section{Introduction}

Pancreatic ductal adenocarcinoma (PDAC) is one of the most fatal cancers worldwide (1). The incidence of PDAC has continuously increased during the past two decades, and its incidence has been reported to have increased from 12.1 per 100,000 in $1997-2000$ to 15.3 per 100,000 in $2013-2016$ (2). It has been reported that PDAC is the second leading cause of cancer-associated mortality worldwide $(3,4)$. Currently, the therapeutic approaches used to treat patients with PDAC are still based on surgical resection, traditional chemotherapy and radiation (5). Despite advancements in these treatment modalities, the survival rate of patients with PDAC remains unsatisfactory with 3-year survival ranging between 16.9 and $25.4 \%$ during 1997-2016 (2,6). Along with the development of molecular science, investigators are now focusing on the molecular genetics of PDAC to identify its subtypes and design precision medicine strategies $(7,8)$. Currently, several genetic variations have been identified in PDAC, such as mutations in the KRAS, cyclin-dependent kinase inhibitor 2A and TP53 genes $(9,10)$. However, the effects of these mutations have been mostly restricted to the research level $(9,11)$. Thus, the application of these findings on treating patients with PDAC is yet to be implemented $(7,8)$.

circular RNAs (circRNAs), characterized by the closed coil structure resulting from a lack of Poly A tail, are a class of small endogenous molecules (12). Despite recent advancements 
in circRNA research, the origin and roles of circRNAs remain unclear. Several biological functions of circRNAs have been established, such as regulating the structure of protein complexes and the expression of their original genes, and sponging microRNAs (miRNAs/miRs) (13-15). With regards to the role of circRNAs in oncology, microarray analyses have indicated that partial circRNAs are aberrantly expressed in different types of cancer, such as lung adenocarcinoma, squamous cell carcinoma and glioma, and involved in their initiation and progression (16-18). Notably, circRNAs exhibit a more stable structure compared with other types of non-coding RNAs in human tissues and cells (19); therefore, several studies have been performed to investigate the expression profiles and the potential functions of circRNAs in patients with cancer $(20,21)$. Previously, two studies analyzed the circRNA expression profiles in patients with PDAC; however, the differentially expressed circRNAs (DEcircRNAs) identified in these studies lacked validation in large scale clinical studies, and their associations with clinical characteristics or survival have not yet been investigated $(22,23)$.

Based on the two datasets from the previous studies, GSE69362 and GSE79634, the present study performed a secondary analysis to determine the candidate circRNAs by identifying the DEcircRNAs with accordant expression trends in the two datasets. A total of nine candidate circRNAs were identified and validated in tissue samples from 60 patients with PDAC via reverse transcription-quantitative (RT-q)PCR analysis. The present study also investigated the correlation of these candidate circRNAs with clinicopathological characteristics, and their association with survival of patients with PDAC.

\section{Materials and methods}

Microarray data collection and analysis. To identify the DEcircRNAs in PDAC, the circRNA expression profile datasets from the National Center of Biotechnology Information Gene Expression Omnibus database were searched (NCBI GEO, http://www.ncbi.nlm.nih.gov/geo). A total of two datasets, GSE69362 and GSE79634, including the circRNA expression profiles in patients with PDAC were screened and downloaded from the GEO database. The GSE69362 dataset included circRNA expression profiles from six PDAC tissues and six paired adjacent normal tissues (24). The GSE79634 dataset included circRNA expression profiles from 20 PDAC tissues and 20 paired adjacent normal tissues (23).

The GEOquery (http://www.bioconductor.org/packages/ release/bioc/html/GEOquery.html) and limma (http://www. bioconductor.org/packages/release/bioc/html/limma.html) packages in R software (version 3.3.2; http://www.r-project.org) were used to process the expression matrix and differential expression analysis, as previously described (25). Principal component analysis (PCA) was performed using the Factoextra package in $\mathrm{R}$ software (version 1.0.7; https://cran.r-project.org/web/ packages/factoextra/index.html). $\log _{2}[$ fold change (FC) $] \geq 11$ and an adjusted $\mathrm{P}$-value $\left(\mathrm{P}_{\mathrm{adj}}\right) \leq 0.05$ were set as thresholds to identify the DEcircRNAs, which were presented as volcano plots using the ggplot2 package (version 3.3.5; https://cran.r-project. org/web/packages/ggplot2/index.html) in R software (version 3.3.2; http://www.r-project.org).
Gene Ontology (GO; http://geneontology.org) and Kyoto Encyclopedia of Genes and Genomes (KEGG; https://www. kegg.jp) enrichment analyses were performed to assess the DEcircRNAs, based on the identified genes. The overlapping upregulated and downregulated DEcircRNAs in both GSE69362 and GSE79634 datasets were displayed using Venn diagrams. A total of nine DEcircRNAs were identified. The circRNA-miRNA network, depicting the interactions between the nine candidate circRNAs and their target miRNAs, was predicted using Miranda software (version 3.3a; https://anaconda.org/bioconda/miranda).

Patients and samples. To further validate the correlation between the expression profiles of the nine candidate circRNAs and the clinicopathological characteristics of patients with PDAC, PDAC and paired adjacent normal tissues were collected from 60 patients with PDAC who underwent surgery between November 2016 and December 2019. Para-cancer tissues were taken $2 \mathrm{~cm}$ away from the tumor margin. There were 36 male patients and 24 female patients. The mean age of patients with PDAC was 63.3 \pm 7.2 years (range, 43-75 years). The surgery for patients with PDAC was performed at Union Hospital, Tongji Medical College, Huazhong University of Science and Technology (Wuhan, China). The inclusion criteria for all patients with PDAC were: i) Pathologically confirmed as PDAC; ii) age $>18$ years; iii) underwent surgical resection; iv) had snap-frozen PDAC and paired adjacent normal tissues; and v) without neoadjuvant therapy. The exclusion criteria were: i) Incomplete clinical data and survival data; and ii) complicated with other malignancies. The PDAC and paired adjacent normal tissues were snap-frozen in liquid nitrogen immediately after surgery. The specimens were stored at $-80^{\circ} \mathrm{C}$. All patients were followed up every 3-6 months by clinic visits or telephone calls. The median follow-up duration was 16.0 months (range, 2.0-36.0 months; between November 2016 and December 2019), and the last follow-up date was December 31, 2019. The present study was approved by the Ethics Committee of Tongji Medical College, Huazhong University of Science and Technology [approval no. (2014), ethics approval no. (S108)]. Written informed consent was provided by all patients or their legal representatives prior to the study start.

Clinical data collection. The clinical data of patients were reviewed, and the clinical characteristics [age, sex, smoking and drinking history, tumor location, pathological grade, tumor size, lymph node metastasis (LNM), T stage, N stage and TNM stage] were recorded. Tumor staging was performed according to the American Joint Committee on Cancer (version 8) TNM staging system (26). Survival data were extracted from the patients' follow-up documents, which were used to measure overall survival (OS) time. Follow-up lasted from November 2016 to December 2019. Patients were followed up every 3-6 months by clinic visits or telephone calls.

Determination of candidate circRNAs. The expression levels of the nine candidate circRNAs were detected in 60 PDAC tissues and paired adjacent normal tissues via RT-qPCR analysis. Total RNA was extracted from PDAC tissues and normal tissues using TRIzol ${ }^{\circledR}$ reagent (Thermo Fisher 
GSE79634 dataset

A

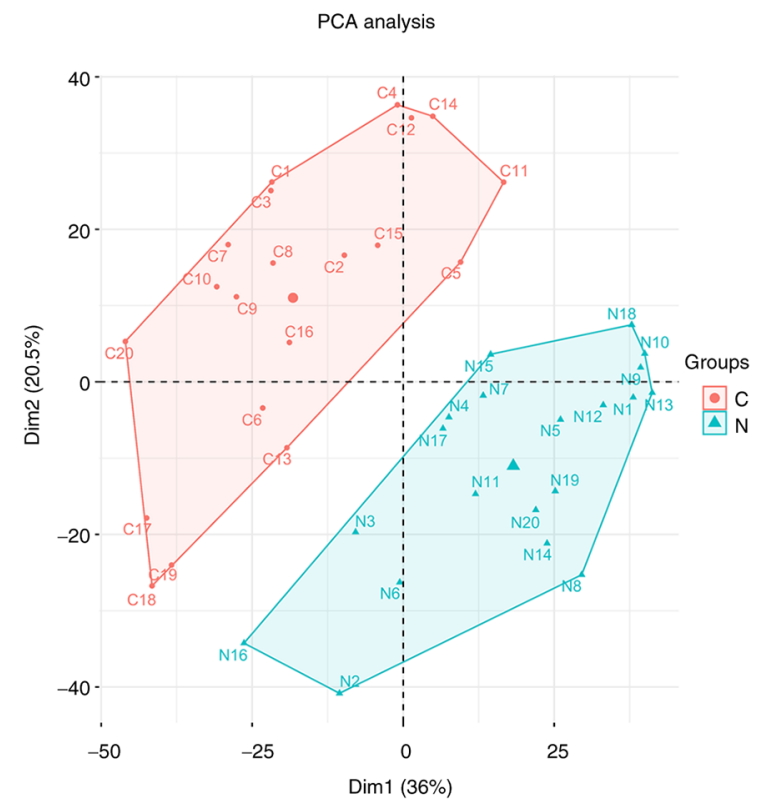

C

GO enrichment analysis

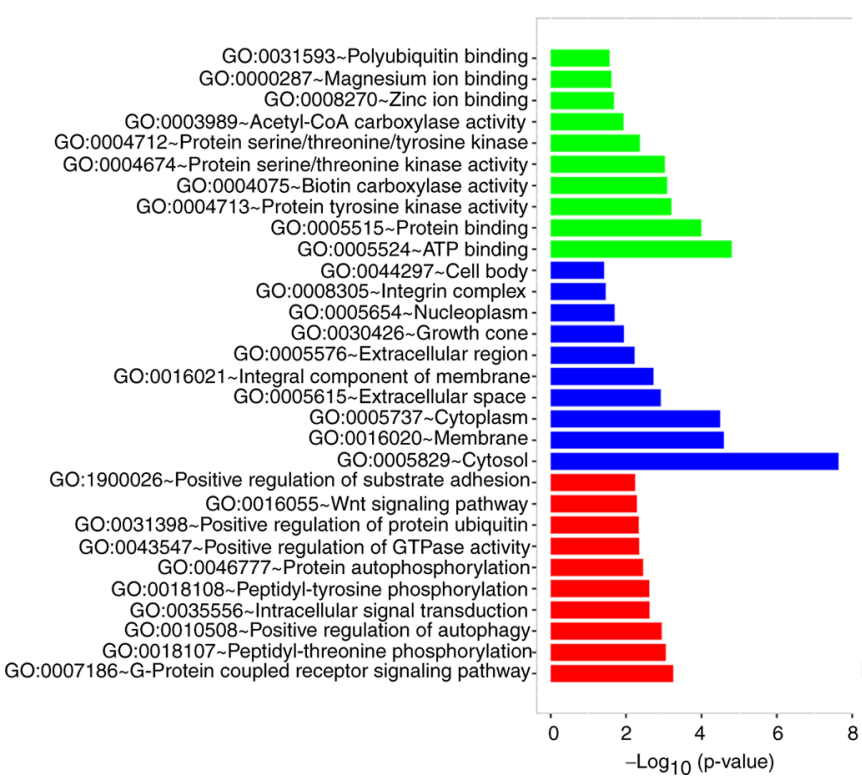

B

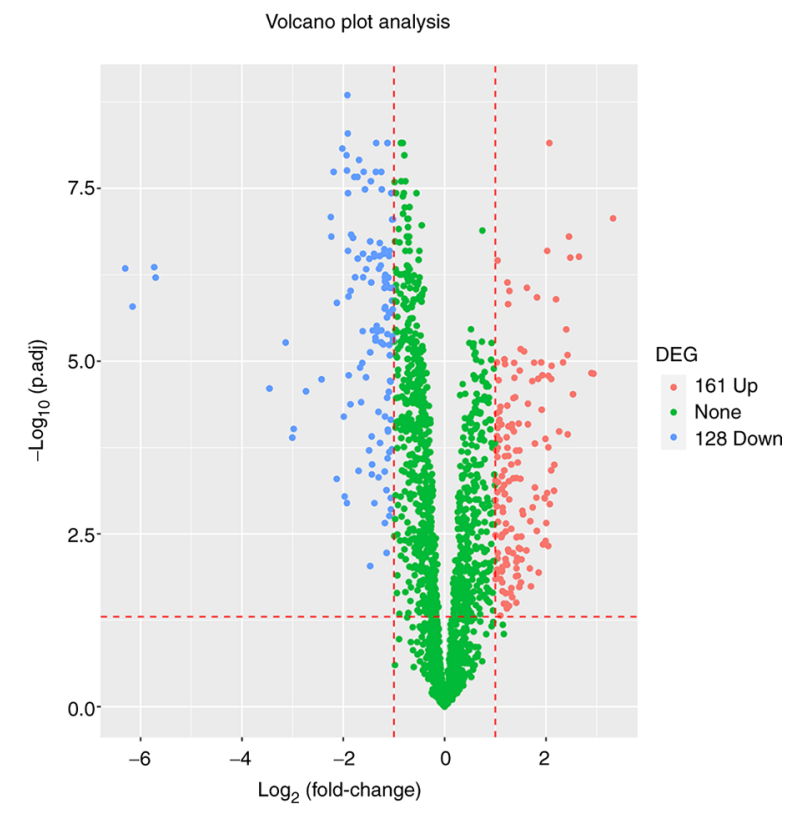

D

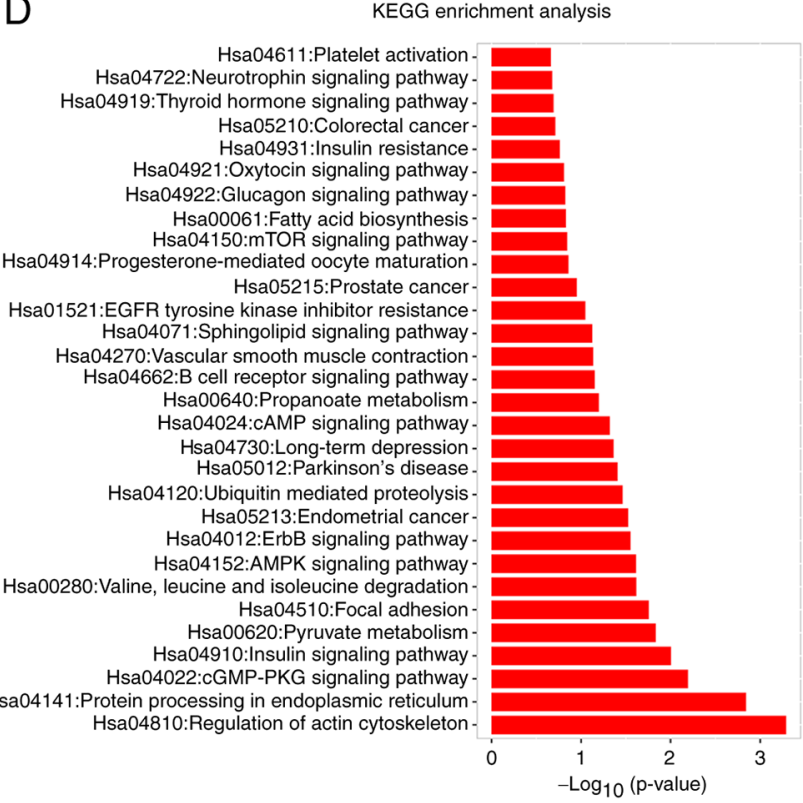

Figure 1. Secondary analyses of the GSE79634 dataset. (A) PCA, (B) volcano plot, (C) GO enrichment (green, molecular function; blue, cellular component; red, biological function) and (D) KEGG enrichment analyses in the GSE79634 dataset, which includes 20 PDAC tissues and 20 paired adjacent normal tissues. PCA, principal component analysis; GO, Gene Ontology; KEGG, Kyoto Encyclopedia of Genes and Genomes; PDAC, pancreatic ductal adenocarcinoma; DEcircRNA, differentially expressed circular RNA; C, cancer; N, normal.

Scientific, Inc.). The linear RNA was subsequently digested using RNase R (Epicentre; Illumina, Inc.) and reverse transcribed into cDNA using the PrimeScript ${ }^{\circledR}$ RT reagent kit (Takara Biotechnology Co., Ltd.). qPCR was subsequently performed using TB Green ${ }^{\circledR}$ Fast qPCR Mix (Takara Biotechnology Co., Ltd.). The primer sequences used for qPCR are listed in Table SI. The specific conditions for RT were as follows: $37^{\circ} \mathrm{C}$ for $15 \mathrm{~min}$ and $85^{\circ} \mathrm{C}$ for $5 \mathrm{sec}$. The thermocycling conditions for qPCR were as follows: Initial denaturation at $95^{\circ} \mathrm{C}$ for $30 \mathrm{sec}$, followed by 40 cycles of $95^{\circ} \mathrm{C}$ for $5 \mathrm{sec}$ and $61^{\circ} \mathrm{C}$ for $10 \mathrm{sec}$. The relative expression was calculated using the $2^{-\Delta \Delta \mathrm{Cq}}$ method (27).

Classification of candidate circRNAs. The candidate circRNAs were classified into high- and low-expression groups according to their median value in the tumor tissue, as follows: 3.345 for circ_0000515, 2.564 for circ_0000517, 2.114 for circ_0000520, 2.999 for circ_0000514, 4.675 for circ_0011385, 1.865 for circ_0055033, 2.395 for circ_0072088, 1.594 for circ_0003528, 1.086 for circ_0008514. 

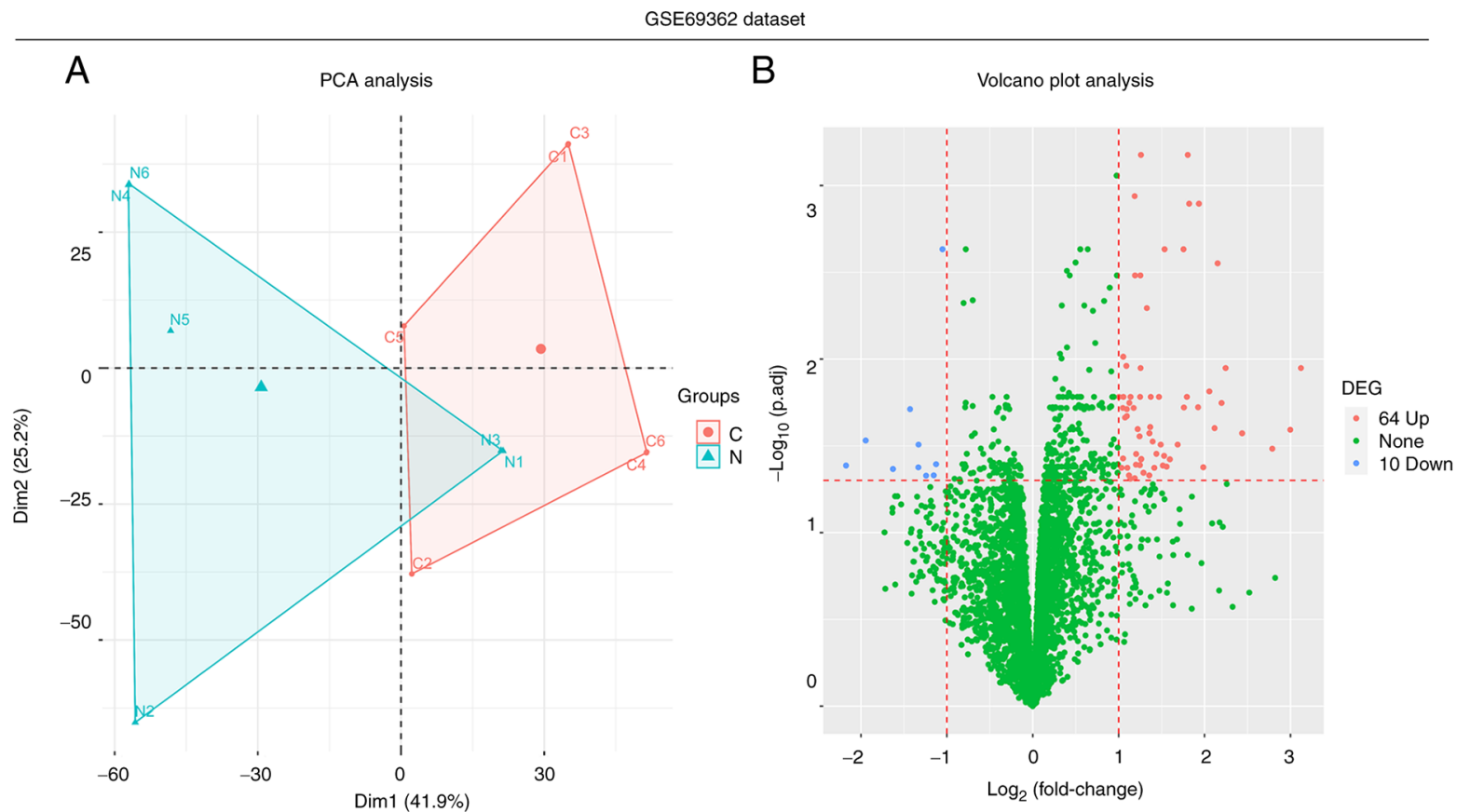

C

GO enrichment analysis

$\mathrm{D}$

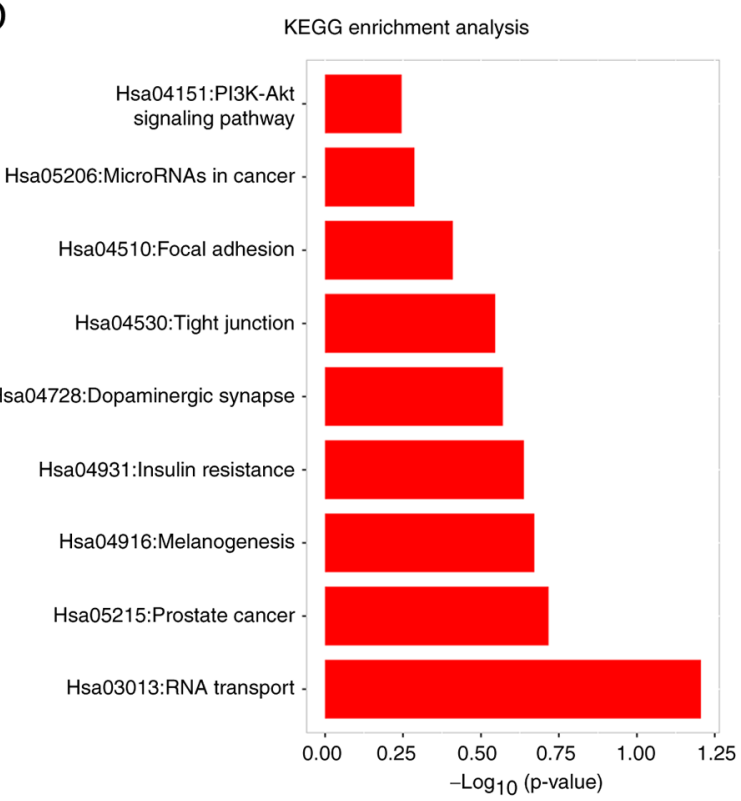

Figure 2. Secondary analyses of the GSE69362 dataset. (A) PCA, (B) volcano plot, (C) GO enrichment and (D) KEGG enrichment analyses in the GSE79634 dataset, which included six PDAC tissues and six paired adjacent normal tissues. PCA, principal component analysis; GO, Gene Ontology; KEGG, Kyoto Encyclopedia of Genes and Genomes; PDAC, pancreatic ductal adenocarcinoma; DEcircRNA, differentially expressed circular RNA; C, cancer; N, normal.

Statistical analysis. Data processing and graphs were plotted using R software (version 3.3.2; http://www.r-project.org), SPSS 22.0 software (IBM Corp.) or GraphPad Prism 7.02 software (GraphPad Software Inc.). Data are presented as the mean \pm SD for continuous data with normal distribution, median with interquartile range for continuous data with skewed distribution and number with percentage for categorical data. Data distribution was determined using the Kolmogorov-Smirnov test. The Wilcoxon signed-rank sum test was used to compare differences in the expression levels of the candidate circRNAs between PDAC tissues and adjacent normal tissues. Receiver operating characteristic (ROC) curve analysis was performed to analyze the performance of the candidate circRNAs in distinguishing between PDAC tissues and adjacent normal tissues. Spearman's rank correlation analysis was performed to assess the correlation between the circRNAs and the clinicopathological characteristics of patients with PDAC. The OS time was measured from the date of surgery to mortality or the last follow-up via the Kaplan-Meier method and log-rank test. $\mathrm{P}<0.05$ was considered to indicate a statistically significant difference.

\section{Results}

circRNA expression profiles and enrichment analysis. In the GSE79634 dataset, PCA analysis revealed that 
A

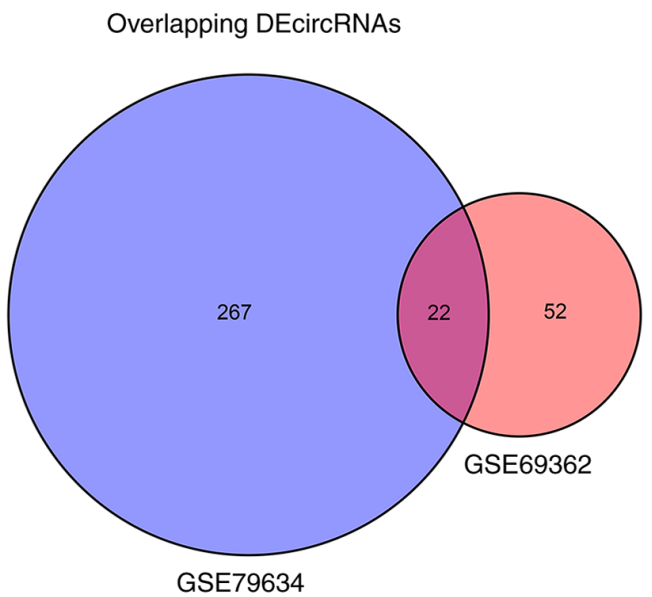

B

Accordant DEcircRNAs

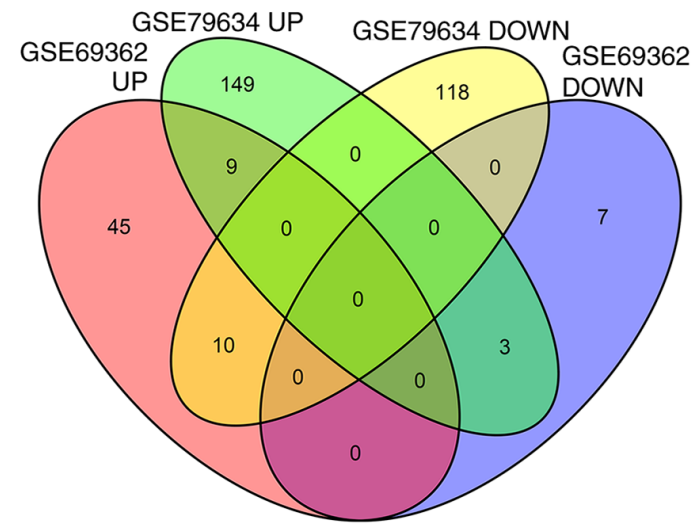

Figure 3. Analyses for accordant DEcircRNAs. (A) The overlapping DEcircRNAs in the GSE79634 and GSE69362 datasets. (B) DEcircRNAs with the same expression trends in both datasets are presented. DEcircRNA, differentially expressed circular RNA.

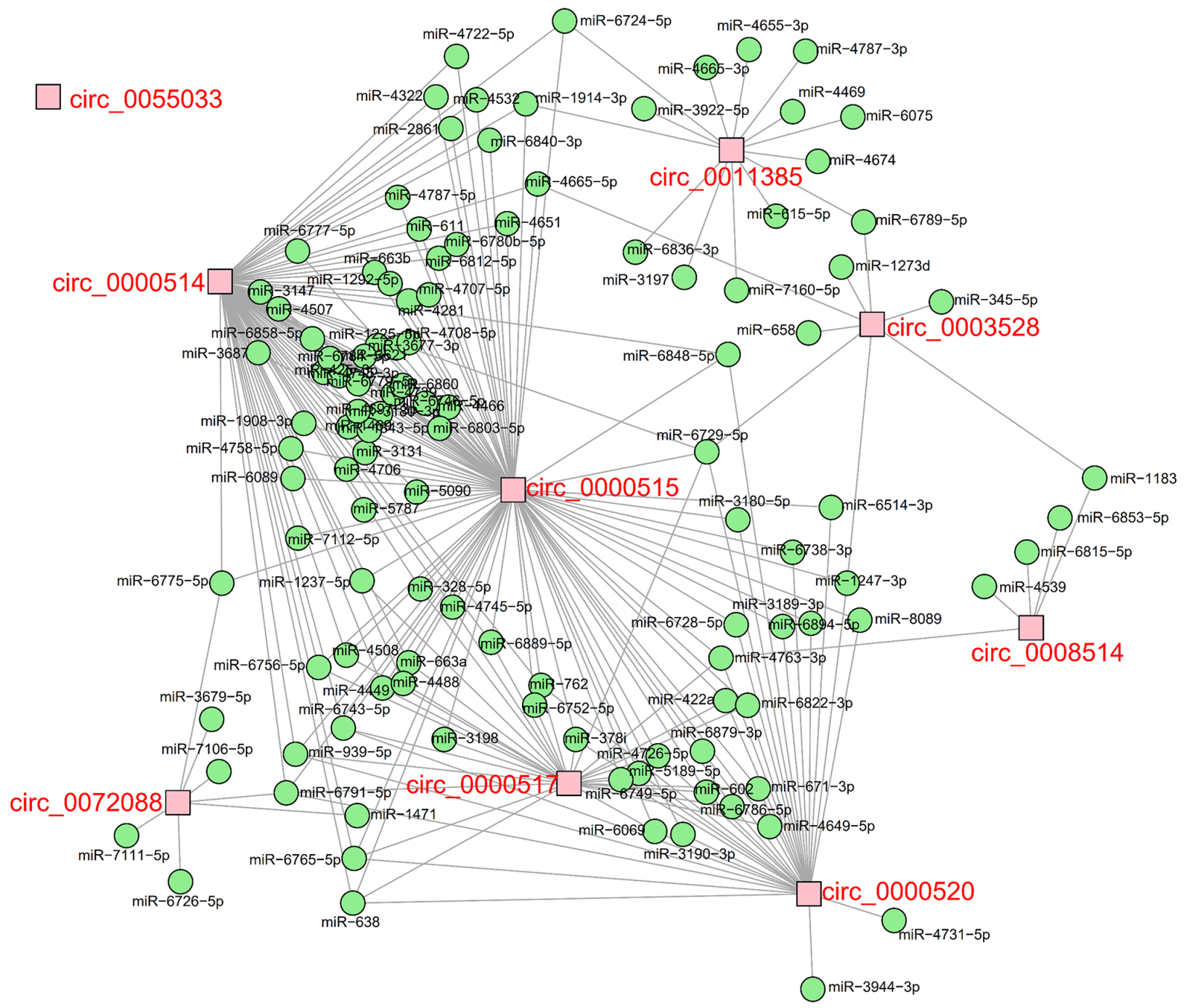

Figure 4. Regulatory network composed of the candidate circRNAs and their targeted miRNAs. Data were obtained from the GSE79634 and GSE69362 datasets. circRNA, circular RNA; miRNA/miR, microRNA.

circRNAs were differentially expressed between 20 PDAC tissues and 20 paired adjacent normal tissues (Fig. 1A). The volcano plot revealed that there were 161 upregulated and
128 downregulated DEcircRNAs in PDAC tissues compared with paired adjacent normal tissues (Fig. 1B). Furthermore, GO enrichment analysis demonstrated that the DEcircRNAs 


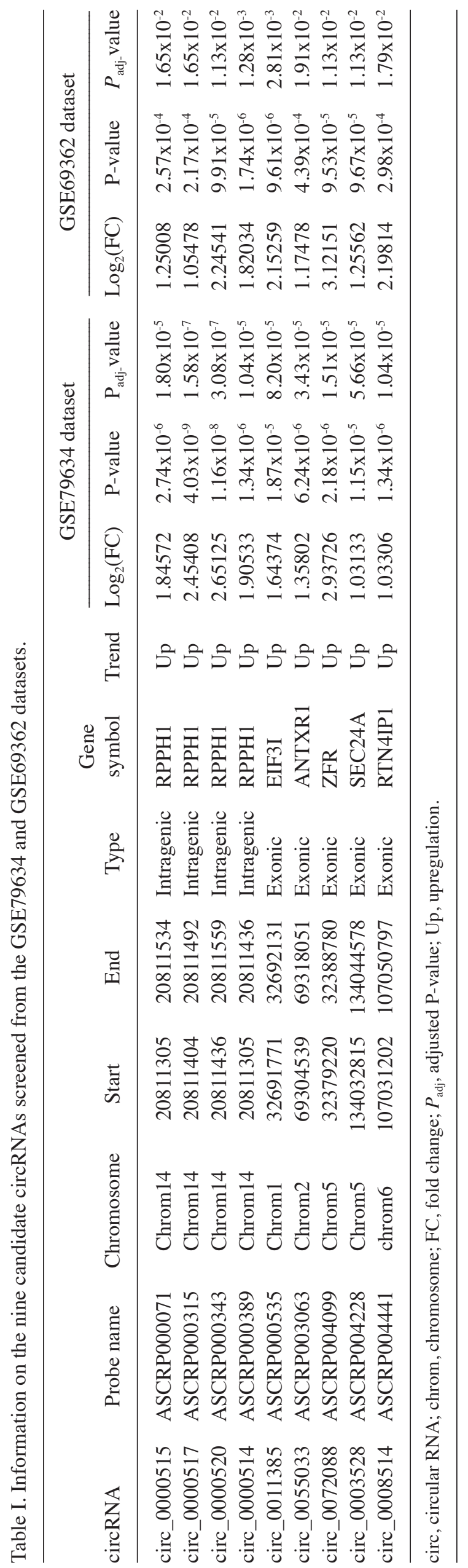

Table II. Clinical characteristics of patients with pancreatic ductal adenocarcinoma $(n=60)$.

\begin{tabular}{|c|c|}
\hline Characteristic & Patient, $\mathrm{n}$ \\
\hline Age, years $($ mean $\pm S D)$ & $63.3 \pm 7.2$ \\
\hline \multicolumn{2}{|l|}{ Sex, n (\%) } \\
\hline Male & $36(60.0)$ \\
\hline Female & $24(40.0)$ \\
\hline \multicolumn{2}{|l|}{ History of smoking, $\mathrm{n}(\%)$} \\
\hline No & $26(43.3)$ \\
\hline Yes & $34(56.7)$ \\
\hline \multicolumn{2}{|l|}{ History of drinking, $\mathrm{n}(\%)$} \\
\hline No & $29(48.3)$ \\
\hline Yes & $31(51.7)$ \\
\hline \multicolumn{2}{|l|}{ Tumor location, $\mathrm{n}(\%)$} \\
\hline Pancreas head & $27(45.0)$ \\
\hline Pancreas body & $21(35.0)$ \\
\hline Pancreas tail & $12(20.0)$ \\
\hline \multicolumn{2}{|l|}{ Pathological grade, n (\%) } \\
\hline G1 & $8(13.4)$ \\
\hline G2 & $26(43.3)$ \\
\hline G3 & $26(43.3)$ \\
\hline Tumor size, $\mathrm{cm}($ mean $\pm \mathrm{SD})$ & $3.8 \pm 1.5$ \\
\hline \multicolumn{2}{|l|}{ LNM, n (\%) } \\
\hline No & $18(30.0)$ \\
\hline Yes & $42(70.0)$ \\
\hline \multicolumn{2}{|l|}{ T stage, n (\%) } \\
\hline $\mathrm{T} 1$ & $9(15.0)$ \\
\hline $\mathrm{T} 2$ & $29(48.3)$ \\
\hline $\mathrm{T} 3$ & $15(25.0)$ \\
\hline $\mathrm{T} 4$ & $7(11.7)$ \\
\hline \multicolumn{2}{|l|}{$\mathrm{N}$ stage, $\mathrm{n}(\%)$} \\
\hline No & $18(30.0)$ \\
\hline N1 & $33(55.0)$ \\
\hline $\mathrm{N} 2$ & $9(15.0)$ \\
\hline \multicolumn{2}{|l|}{ TNM stage, $\mathrm{n}(\%)$} \\
\hline IA & $4(6.7)$ \\
\hline IB & $4(6.7)$ \\
\hline IIA & $8(13.3)$ \\
\hline IIB & $28(46.7)$ \\
\hline III & $16(26.6)$ \\
\hline
\end{tabular}

LNM, lymph node metastasis; TNM, tumor-node-metastasis.

were predominantly enriched in the 'ATP binding' in terms of molecular function, the 'cytosol' in terms of cellular component and the 'G-protein coupled receptor signaling pathway' in terms of biological function (Fig. 1C). In addition, the most significantly enriched pathways in the KEGG enrichment analysis associated with the candidate DEcircRNAs were the 'regulation of actin cytoskeleton', 'protein processing in endoplasmic reticulum', 'cGMP-PKG signaling' and 'insulin signaling' pathways (Fig. 1D). These results suggested that the 
A

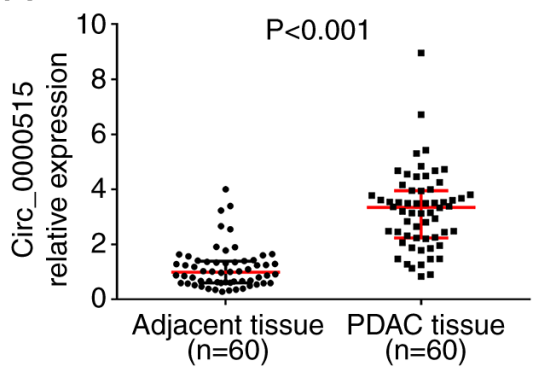

$\mathrm{D}$

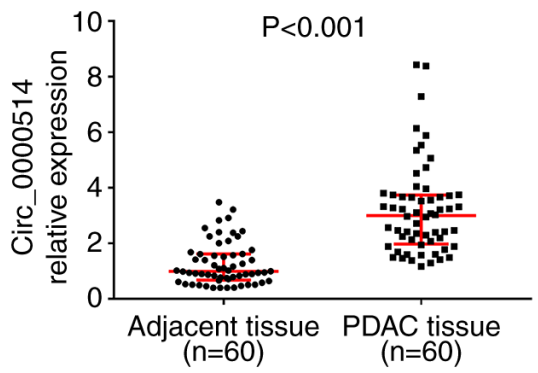

G

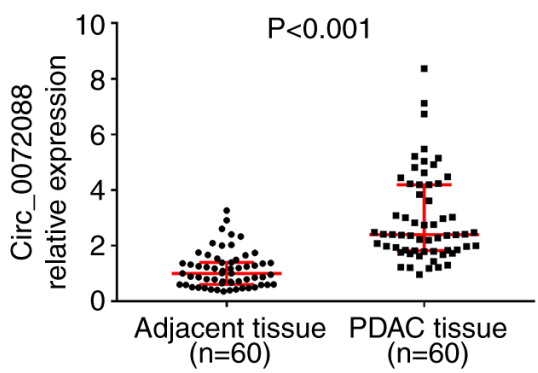

B

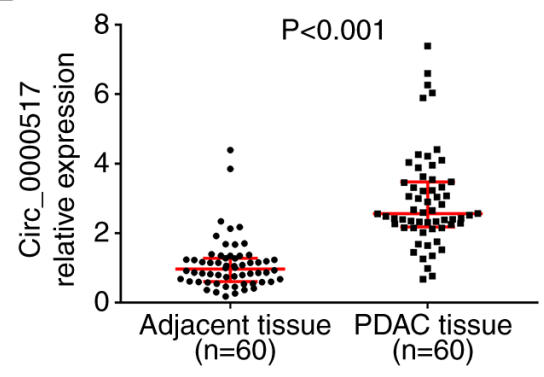

E

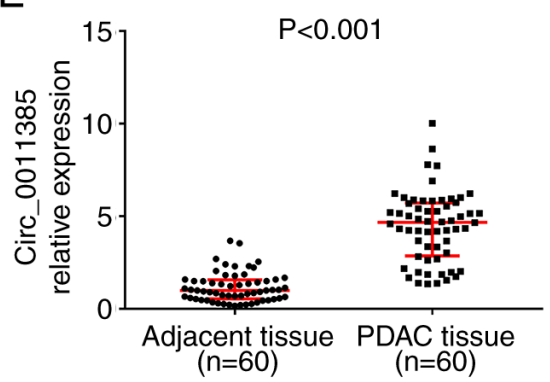

$\mathrm{H}$

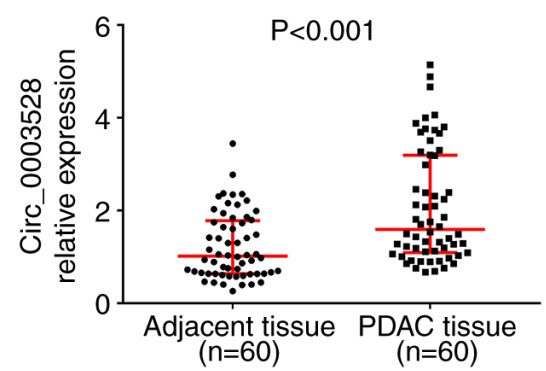

C

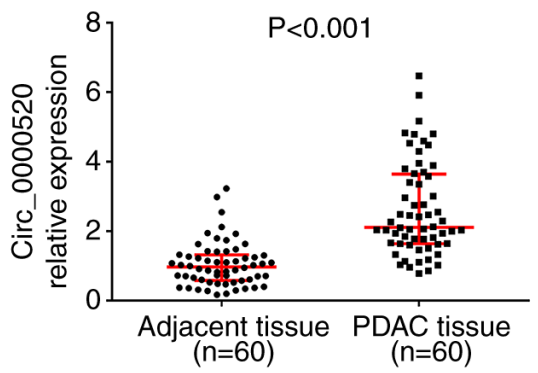

F

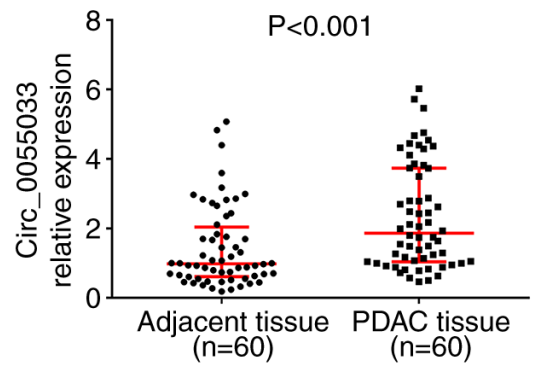

I

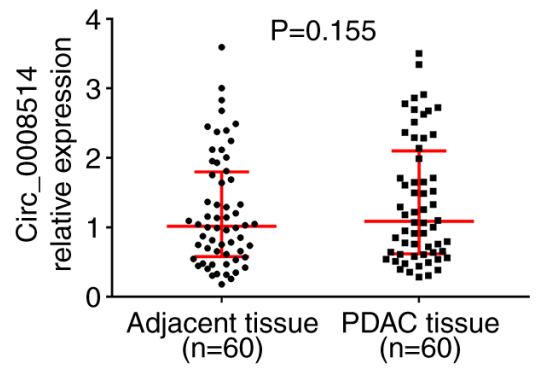

Figure 5. Expression levels of the candidate circRNAs between PDAC tissues and paired adjacent normal tissues. The expression levels of (A) circ_0000515, (B) circ_0000517, (C) circ_0000520, (D) circ_0000514, (E) circ_0011385, (F) circ_0055033, (G) circ_0072088, (H) circ_0003528 and (I) circ_0008514 between PDAC tissues and paired adjacent normal tissues. circRNA, circular RNA; PDAC, pancreatic ducal adenocarcinoma.

circRNAs in the GSE79634 dataset were differently expressed between the tumor tissue and adjacent tissue with 161 upregulated and 128 downregulated DEcircRNAs, and they may be involved in several cellular process.

In the GSE69362 dataset, PCA analysis revealed that circRNAs were differentially expressed between six PDAC tissues and six paired adjacent normal tissues (Fig. 2A). The volcano plot revealed that there were 64 upregulated and 10 downregulated DEcircRNAs in PDAC tissues compared with normal tissues (Fig. 2B). Furthermore, GO enrichment analysis demonstrated that the DEcircRNAs were predominantly enriched in the 'zinc ion binding' in terms of molecular function, the 'cytoplasm' in terms of cellular component and the 'mitotic nuclear envelope disassembly' in terms of biological process (Fig. 2C). In addition, the most significantly enriched pathways in the KEGG enrichment analysis associated with the candidate DEcircRNAs were the 'RNA transport', 'prostate cancer', 'melanogenesis' and 'insulin resistance' signaling pathways (Fig. 2D). These results suggested that the circRNAs in the GSE69362 dataset were differently expressed between the tumor tissue and adjacent tissue with 64 upregulated and 10 downregulated DEcircRNAs, and they might be involved in several cellular process.
Selection of candidate circRNAs. Further analysis revealed that 22 DEcircRNAs were overlapped between the GSE79634 and GSE69362 datasets (Fig. 3A). Among the overlapping DEcircRNAs, nine were upregulated in PDAC tissues compared with paired adjacent normal tissues in both datasets (Fig. 3B). These nine DEcircRNAs were selected as candidate circRNAs and their target miRNAs were predicted. The regulatory network of the DEcircRNAs and their target miRNAs is presented in Fig. 4. The information of these nine candidate circRNAs is presented in Table I, which includes the probe name, located chromosome, start, end, type, gene symbol, expression trend, $\log _{2}(\mathrm{FC}), \mathrm{P}$-value and $\mathrm{P}_{\mathrm{adj}}$-value in the GSE79634 and GSE69362 datasets. These results suggested that 9 upregulated in tumor tissue, overlapping DEcircRNAs between GSE79634 and GSE69362 datasets were identified as candidate circRNAs.

Characteristics of patients with PDAC. Among the 60 patients with PDAC, $36(60.0 \%)$ were men and $24(40.0 \%)$ were women, with a mean age of $63.3 \pm 7.2$ years, and the number of patients with tumor location at pancreas head, pancreas body and pancreas tail was 27 (45.0\%), 21 (35.0\%) and $12(20.0 \%)$, respectively. The number of patients with G1, G2 and G3 
A
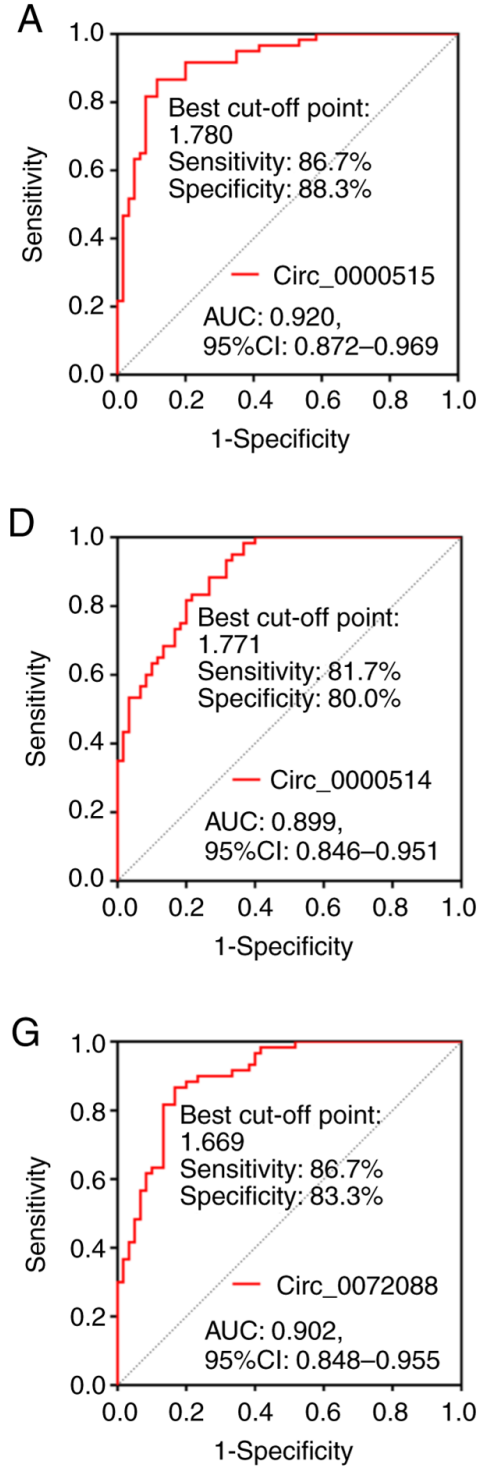
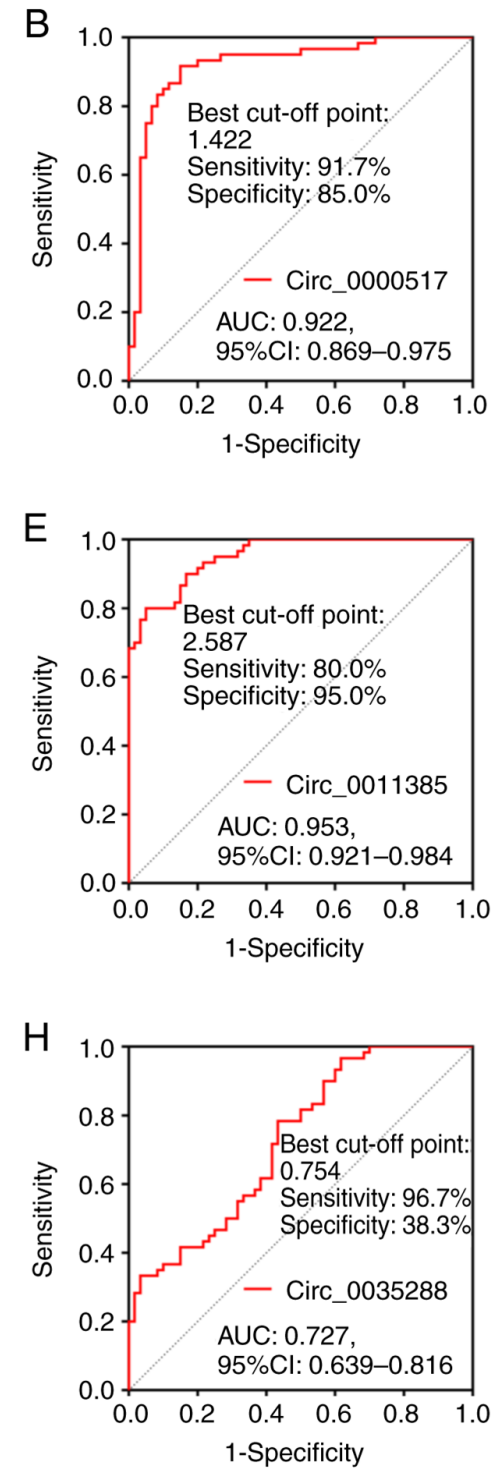
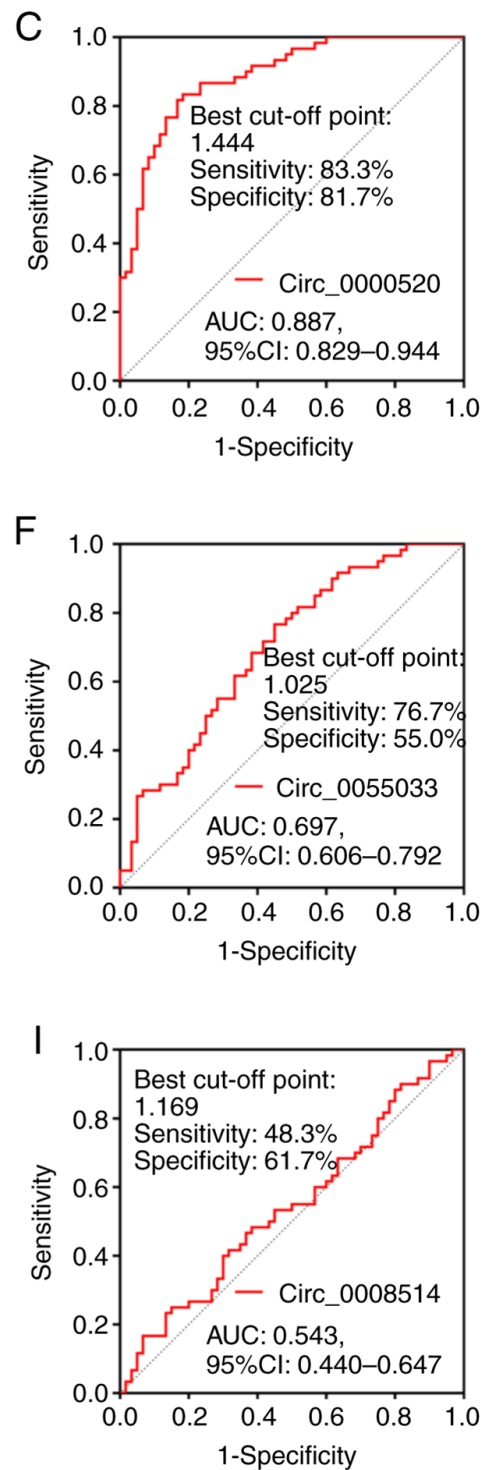

Figure 6. ROC curve analysis of the candidate circRNAs for distinguishing PDAC tissues from paired adjacent normal tissues. ROC curve analysis was performed to determine the AUC values of (A) circ_0000515, (B) circ_0000517, (C) circ_0000520, (D) circ_0000514, (E) circ_0011385, (F) circ_0055033, (G) circ_0072088, (H) circ_0003528 and (I) circ_0008514 for distinguishing PDAC tissues from paired adjacent normal tissues. ROC, receiver operating characteristic; circRNA, circular RNA; PDAC, pancreatic ductal adenocarcinoma; AUC, area under the curve; CI, confidence interval.

pathological grade was $8(13.3 \%), 26(43.3 \%)$ and $26(43.3 \%)$, respectively. The mean tumor size was $3.8 \pm 1.5 \mathrm{~cm}$. A total of 42 patients $(70.0 \%)$ had PDAC LNM. In addition, the number of patients with IA, IB, IIA, IIB and III TNM stage was 4 (6.7\%), 4 (6.7\%), 8 (13.3\%), 28 (46.7\%) and 16 (26.7\%), respectively. All clinicopathological characteristics are presented in Table II. These results indicated the characteristics of patients with PDAC enrolled in the present study.

Candidate circRNA expression levels in patients with PDAC. Among the nine candidate circRNAs, the expression levels of circ_0000515 ( $\mathrm{P}<0.001$; Fig. 5A), circ_0000517 ( $\mathrm{P}<0.001$; Fig. 5B), circ_0000520 ( $\mathrm{P}<0.001$; Fig. 5C), circ-0000514 $(\mathrm{P}<0.001$; Fig. 5D), circ_0011385 ( $\mathrm{P}<0.001 ;$ Fig. 5E), circ_0055033 ( $\mathrm{P}<0.001$; Fig. 5F), circ_0072088 $(\mathrm{P}<0.001$; Fig. 5G) and circ_0003528 ( $\mathrm{P}<0.001$; Fig. 5H) were significantly upregulated in PDAC tissues compared with paired adjacent normal tissues. However, circ_0008514 expression
$(\mathrm{P}=0.155$; Fig. 5I) was not significantly different between PDAC tissues and paired adjacent normal tissues. Furthermore, ROC curve analysis demonstrated that circ_0000515 (Fig. 6A), circ_0000517 (Fig.6B), circ_0000520 (Fig.6C), circ_0000514 (Fig. 6D), circ_0011385 (Fig. 6E) and circ_0072088 (Fig. 6G) exhibited good area under the curve (AUC) values to distinguish PDAC tissues from paired adjacent normal tissues. The AUC values for these circRNAs were 0.920, 0.922, 0.887, $0.899,0.953$ and 0.902 , respectively. Conversely, circ-0055033 (Fig. 6F) and circ_0003528 (Fig. 6H) exhibited ordinary values in differentiating PDAC tissues from paired adjacent normal tissues, with AUC values of 0.697 and 0.727 , respectively. Notably, circ_0008514 (Fig. 6I) failed to distinguish between PDAC tissues and paired adjacent normal tissues. These findings suggested that eight candidate circRNAs were highly expressed in PDAC tissues compared with paired adjacent normal tissues and they could distinguish PDAC tissues from paired adjacent normal tissues. 


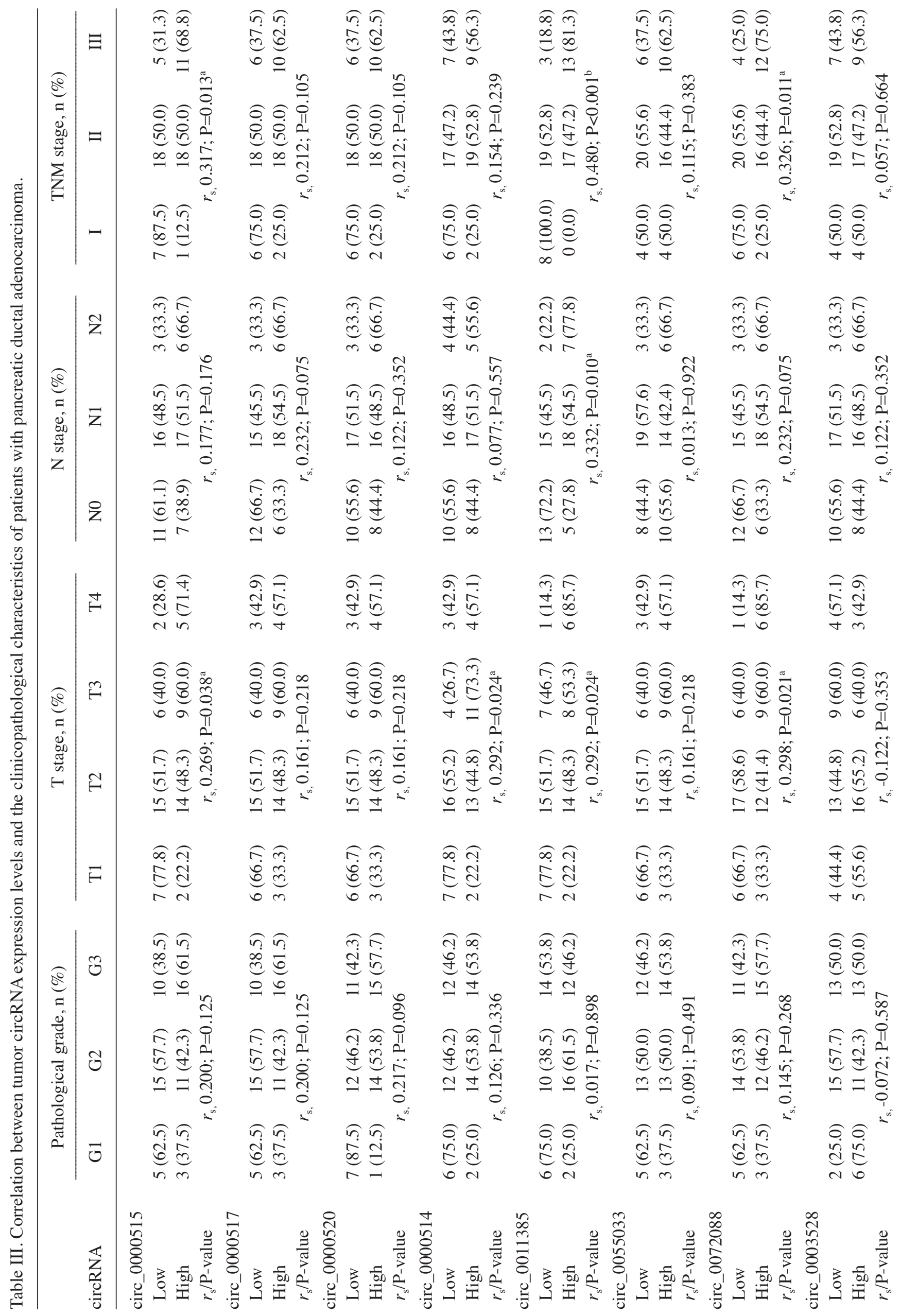


Correlation between the expression levels of the candidate circRNAs and the clinicopathological characteristics of patients with PDAC. Spearman's rank correlation analysis was performed to assess the correlation between the circRNAs and the clinicopathological characteristics of patients with PDAC. The results demonstrated that tumor circ_0000515 expression was positively correlated with $\mathrm{T}$ stage $(\mathrm{P}=0.038)$ and TNM stage $(\mathrm{P}=0.013)$ in patients with PDAC. In addition, tumor circ_0000514 expression was positively correlated with T stage $(\mathrm{P}=0.024)$, while tumor circ_0011385 expression was positively correlated with $\mathrm{T}$ stage $(\mathrm{P}=0.024), \mathrm{N}$ stage $(\mathrm{P}=0.010)$ and TNM stage $(\mathrm{P}<0.001)$. Furthermore, tumor circ_0072088 expression was positively correlated with $\mathrm{T}$ stage $(\mathrm{P}=0.021)$ and TNM stage $(\mathrm{P}=0.011)$ in patients with PDAC (Table III). The aforementioned findings mentioned demonstrated that four candidate circRNAs were associated with aggravating clinicopathological characteristics of patients with PDAC.

Association between the expression levels of the candidate circRNAs and OS time in patients with PDAC. The OS time was significantly shorter in patients with high circ_0000515 expression than those with low circ_0000515 expression $(\mathrm{P}=0.013$; Fig. 7A). Furthermore, OS time was significantly shorter in patients with high circ_0011385 expression than those with low circ_0011385 expression ( $\mathrm{P}=0.014$; Fig. 7E). However, the expression levels of circ_0000517 ( $\mathrm{P}=0.128$; Fig. 7B), circ_0000520 ( $\mathrm{P}=0.163$; Fig. 7C), circ_0000514 $(\mathrm{P}=0.135$; Fig. 7D), circ_0055033 ( $\mathrm{P}=0.184$; Fig. 7F), circ_0072088 $(\mathrm{P}=0.271$; Fig. 7G), circ_0003528 ( $\mathrm{P}=0.667$; Fig. 7H) and circ_0008514 ( $\mathrm{P}=0.862$; Fig. 7I) in PDAC tissues were not associated with OS time. As aforementioned, high levels of these two candidate circRNAs (circ_0000515 high and circ_0011385 high) were associated with shorter OS times.

RT-qPCR analysis demonstrated that among the nine candidate circRNAs in patients with PDAC, eight candidate circRNAs were markedly dysregulated in PDAC tissues compared with paired adjacent normal tissues; four were correlated with tumor stages and two were associated with OS in patients with PDAC (Table IV). Notably, the expression levels of circ_0000515 and circ_0011385 were upregulated in PDAC tissues compared with paired adjacent normal tissues, and were correlated with tumor stage, and associated with OS of patients with PDAC. Thus, these two candidate circRNAs may be used as potential biomarkers in the treatment of PDAC.

\section{Discussion}

The present study performed a secondary analysis based on datasets from two previous studies, namely GSE79634 and GSE69362, and candidate circRNAs were identified among the DEcircRNAs $(22,28)$. The expression levels of the candidate circRNAs and their correlation with the clinicopathological characteristics of patients with PDAC were evaluated in a larger sample size. It was revealed that nine circRNAs exhibited the same expression profile in both datasets. These circRNAs were selected as candidate circRNAs and their expression profile in tissue samples from 60 patients with PDAC was assessed via RT-qPCR analysis. The results demonstrated that 8/9 candidate circRNAs exhibited differentiated expression 
Table IV. Summary of study findings.

\begin{tabular}{|c|c|c|c|c|c|c|}
\hline \multirow[b]{2}{*}{ circRNA } & \multirow{2}{*}{$\frac{\text { Significant difference }}{\text { PDAC vs. adjacent }}$} & \multicolumn{5}{|c|}{ Significant correlation } \\
\hline & & Pathological grade & T stage & $\mathrm{N}$ stage & TNM stage & OS \\
\hline circ_0000515 & Yes & No & Yes & No & Yes & Yes \\
\hline circ_0000517 & Yes & No & No & No & No & No \\
\hline circ_0000520 & Yes & No & No & No & No & No \\
\hline circ_0000514 & Yes & No & Yes & No & No & No \\
\hline circ_0011385 & Yes & No & Yes & Yes & Yes & Yes \\
\hline circ_0055033 & Yes & No & No & No & No & No \\
\hline circ_0072088 & Yes & No & Yes & No & Yes & No \\
\hline circ_0003528 & Yes & No & No & No & No & No \\
\hline circ_0008514 & No & No & No & No & No & No \\
\hline
\end{tabular}

circ, circular RNA; PDAC, pancreatic ductal adenocarcinoma; TNM, tumor-node-metastasis; OS, overall survival.

A

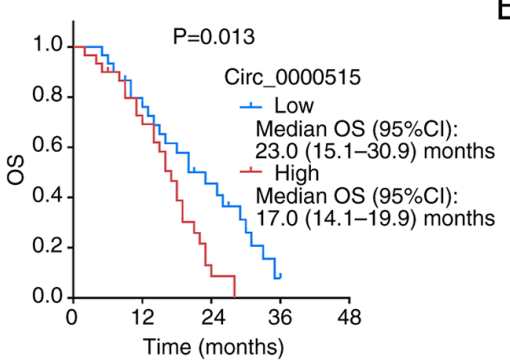

D

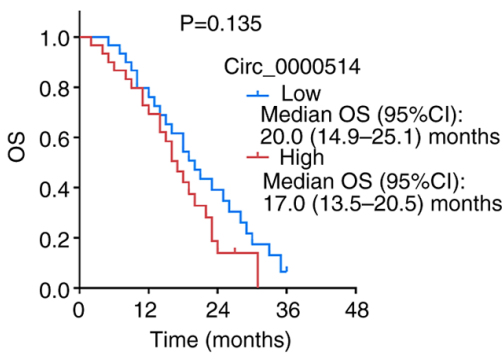

G

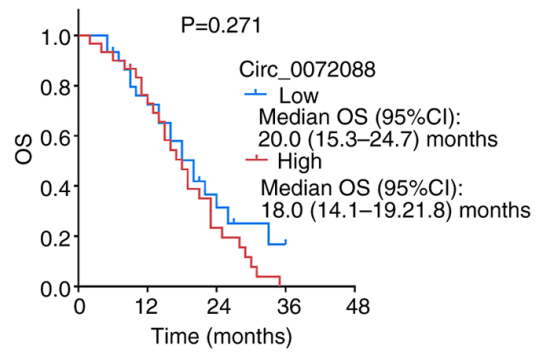

B

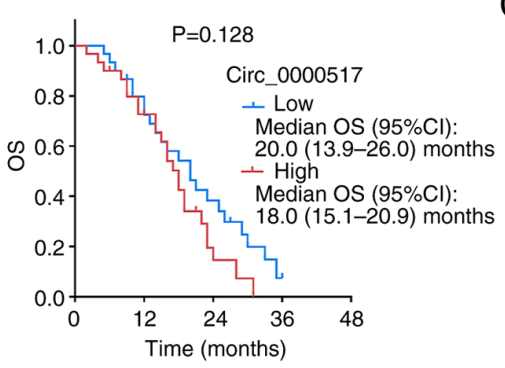

E

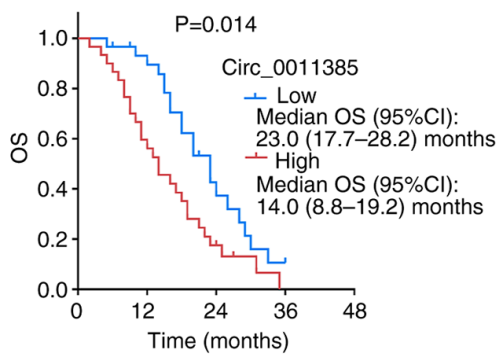

$\mathrm{H}$

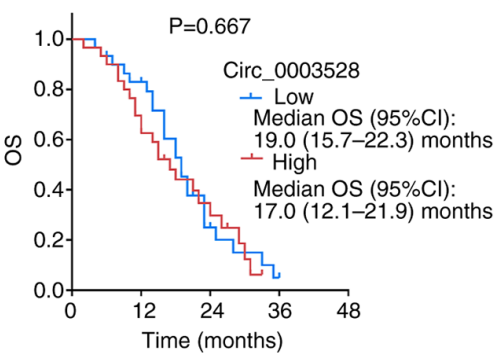

I
C

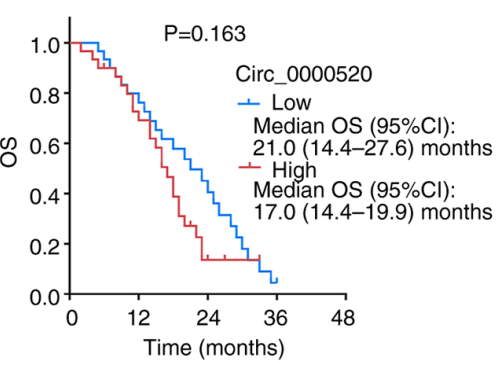

$\mathrm{F}$
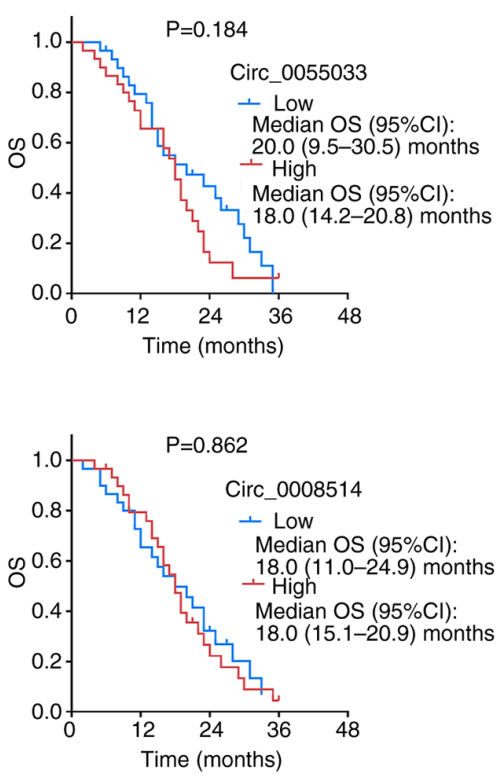

Figure 7. OS analysis between patients with low and high expression levels of the candidate circRNAs. The association between the expression levels of (A) circ_0000515, (B) circ_0000517, (C) circ_0000520, (D) circ_0000514, (E) circ_0011385, (F) circ_0055033, (G) circ_0072088, (H) circ_0003528 and (I) circ_0008514 and OS time in patients with pancreatic ductal adenocarcinoma. OS, overall survival; circRNA, circular RNA; CI, confidence interval.

profiles in tumor tissues compared with paired adjacent normal tissues. Furthermore, five candidate circRNAs were positively correlated with tumor stage, and two (circ_0000515 and circ_0011385) were negatively associated with OS in patients with PDAC. Notably, circ_0000515 and circ_0011385 were not only highly expressed in PDAC tissues compared with normal tissues, but were also correlated with more advanced TNM stage and shorter survival time in patients with PDAC.

The roles of circRNAs in PDAC remain unclear, unlike in other solid tumors, such as gastric carcinoma and 
nasopharyngeal carcinoma $(29,30)$. A previous study reported that circ-ASH2-like histone lysine methyltransferase complex subunit (ASH2L) is upregulated in PDAC cells, which enhances PDAC cell proliferation, migration and invasion capacities (31). Another study demonstrated that circ-bifunctional apoptosis regulator (BFAR) elevates the proliferation, migration and invasion of PDAC cells, and promotes the growth and metastasis of tumors in PDAC animal models (32). Furthermore, circ-BFAR can positively regulate the expression levels of epithelial-to-mesenchymal transition markers by mediating the miR-34b-5p/mesenchymal-to-epithelial transition factor/protein kinase B (Akt) axis in PDAC cells (32). Only a few studies have investigated the correlation between the expression of circRNAs and the clinicopathological characteristics of patients with PDAC $(33,34)$. However, a previous study reported that decreased circ_0001649 expression in PDAC tissues is associated with low tumor stage and increased differentiation level in patients (35). Taken together, these studies suggest that certain circRNAs are associated with disease progression and may serve as potential biomarkers in PDAC.

The present study identified nine circRNAs presenting with the same expression trends in the two datasets (GSE79634 and GSE69362), which were selected as candidate circRNAs for RT-qPCR analyses in a cohort of 60 patients with PDAC. The results demonstrated that $8 / 9$ candidate circRNAs were notably upregulated in PDAC tissues compared with paired adjacent normal tissues, and five of them were positively associated with TNM stage in patients with PDAC. This may be due to that these circRNAs may promote the malignant behaviors of PDAC cells by interacting with other tumor-related factors or signaling pathways, which in turn can promote tumor progression, eventually leading to a more advanced tumor stage in patients with PDAC. For instance, as reported by a previous study about other circRNAs in PDAC, these circRNAs might sponge miRNAs to enhance PDAC cell proliferation, invasion and migration (32). However, regarding the five candidate circRNAs identified in the present study, no mechanistic studies have been reported to date; therefore, in vivo and in vitro studies are urgently required to verify their effects on PDAC.

In the present study, two candidate circRNAs, circ_0000515 and circ_0011385, were not only positively associated with TNM stage, but also negatively associated with OS time in patients with PDAC. Thus, it was hypothesized that these two candidate circRNAs may serve as prognostic factors in PDAC management. To the best of our knowledge, the present study was the first to report the potential role of these two circRNAs as biomarkers in PDAC. Similarly, other circRNAs, including circ_0030235 and circ_0007534, have been identified as prognostic biomarkers of PDAC (33-36). A previous study suggested that circ-ASH2L may serve as a prognostic biomarker in patients with PDAC as its high expression in PDAC tissues was associated with poor survival outcomes (31). Another study reported that circ_0001649 expression is downregulated in PDAC tissues compared with normal tissues, and multivariate regression analysis revealed that its expression may be an independent prognostic factor for survival (37). A previous study demonstrated that circ_0030235 expression is upregulated in PDAC tissues, and its expression is associated with poor prognosis (35). In terms of the circRNAs identified in the present study, circ_0000515 and circ_0011385 have not yet been identified in PDAC. However, they have been reported in different types of cancer. For example, an in vivo and in vitro study revealed that circ_0000515 enhances progression of cervical cancer by sponging miR-326 expression via increasing E-twenty six transcription factor ELK1 expression (38). Another study reported that circ_0011385 promotes thyroid cancer development by sponging miR-361-3p expression in vitro and in vivo (39). The results of the present study may be explained as follows: Several target miRNAs of these two candidate circRNAs function as antitumor factors in different types of cancer. For example, the target miRNA of circ_0000515, miR-939, and the target miRNA of circ_0011385, miR-615, are both considered tumor suppressors that inhibit cancer cell invasion and migration in various types of cancer (40-43). Thus, it was hypothesized that circ_0000515 and circ_0011385 may potentially function as regulators in enhancing the malignant behavior of PDAC by sponging their target miRNAs, which promotes the tumor progression of PDAC. In addition, circ_0000515 and circ_0011385 may serve as biomarkers for PDAC management in clinical practice.

The present study is not without limitations. First, the statistical power can be diminished due to the relatively small sample size of 60 patients with PDAC. Secondly, the molecular functions of the candidate circRNAs in PDAC were not investigated in the present study, which should be validated by in vitro and in vivo experiments. Thirdly, microarray analysis may present some bias (44); however, the present study performed RT-qPCR analysis to validate the expression trends of the candidate circRNAs in patients with PDAC. Furthermore, a control group of healthy individuals was not included in the present study. However, it was difficult to acquire pancreatic tissue samples from healthy individuals. Thus, prospective studies will aim to include this control cohort. Fourthly, the expression levels of the circRNAs were only assessed in tissue samples but not peripheral samples, which is inconvenient for clinical application. This was the case as circRNAs are abundantly expressed in tissue samples compared with peripheral blood $(19,45)$.

In conclusion, the results of the present study provide evidence that circ_0000515 and circ_0011385 may serve as biomarkers for disease control and determine the prognosis of patients with PDAC, which implies their potential for guiding the management of patients with PDAC.

\section{Acknowledgements}

Not applicable.

\section{Funding}

No funding was received.

\section{Availability of data and materials}

The datasets used and/or analyzed during the current study are available from the corresponding author upon reasonable request. 


\section{Authors' contributions}

YX conceived, designed and supervised the present study, and revised the manuscript for important intellectual content. HW performed the experiments and provided technical support. BW and LW analyzed the data and drafted the initial manuscript. YX and HW confirm the authenticity of all the raw data. All authors have read and approved the final manuscript.

\section{Ethics approval and consent to participate}

The present study was approved by the Ethics Committee of Tongji Medical College, Huazhong University of Science and Technology [approval number (2014), ethics approval no. (S108)]. Written informed consent was provided by all patients or their legal representatives prior to the study commencement.

\section{Patient consent for publication}

Not applicable.

\section{Competing interests}

The authors declare that they have no competing interests.

\section{References}

1. Storz P and Crawford HC: Carcinogenesis of pancreatic ductal adenocarcinoma. Gastroenterology 158: 2072-2081, 2020.

2. Latenstein AEJ, van der Geest LGM, Bonsing BA, Groot Koerkamp B, Haj Mohammad N, de Hingh IHJT, de Meijer VE, Molenaar IQ, van Santvoort HC, van Tienhoven G, et al: Nationwide trends in incidence, treatment and survival of pancreatic ductal adenocarcinoma. Eur J Cancer 125: 83-93, 2020.

3. Rahib L, Smith BD, Aizenberg R, Rosenzweig AB, Fleshman JM and Matrisian LM: Projecting cancer incidence and deaths to 2030: The unexpected burden of thyroid, liver, and pancreas cancers in the United States. Cancer Res 74: 2913-2921, 2014.

4. Siegel RL, Miller KD and Jemal A: Cancer statistics, 2017. CA Cancer J Clin 67: 7-30, 2017.

5. Winter K, Talar-Wojnarowska R, Dąbrowski A, Degowska M, Durlik M, Gąsiorowska A, Głuszek S, Jurkowska G, Kaczka A, Lampe $\mathrm{P}$, et al: Diagnostic and therapeutic recommendations in pancreatic ductal adenocarcinoma. Recommendations of the working group of the Polish pancreatic club. Prz Gastroenterol 14: $1-18,2019$.

6. Sarantis P, Koustas E, Papadimitropoulou A, Papavassiliou AG and Karamouzis MV: Pancreatic ductal adenocarcinoma: Treatment hurdles, tumor microenvironment and immunotherapy. World J Gastrointest Oncol 12: 173-181, 2020.

7. Rishi A, Goggins M, Wood LD and Hruban RH: Pathological and molecular evaluation of pancreatic neoplasms. Semin Oncol 42: 28-39, 2015.

8. Hackeng WM, Hruban RH, Offerhaus GJ and Brosens LA: Surgical and molecular pathology of pancreatic neoplasms. Diagn Pathol 11: 47, 2016.

9. Vivekanandhan S, Madamsetty VS, Angom RS, Dutta SK, Wang E, Caulfield T, Pletnev AA, Upstill-Goddard R, Asmann YW, Chang D, et al: Role of PLEXIND1/TGF $\beta$ signaling axis in pancreatic ductal adenocarcinoma progression correlates with the mutational status of KRAS. Cancers (Basel) 13: 4048, 2021.

10. Sinn M, Sinn BV, Treue D, Keilholz U, Damm F, Schmuck R, Lohneis P, Klauschen F, Striefler JK, Bahra M, et al: TP53 mutations predict sensitivity to adjuvant gemcitabine in patients with pancreatic ductal adenocarcinoma: Next-generation sequencing results from the CONKO-001 trial. Clin Cancer Res 26: 3732-3739, 2020.
11. Gao J, Chen X, Li X, Miao F, Fang W, Li B, Qian X and Lin X: Differentiating TP53 mutation status in pancreatic ductal adenocarcinoma using multiparametric MRI-derived radiomics. Front Oncol 11: 632130, 2021.

12. Eger N, Schoppe L, Schuster S, Laufs U and Boeckel JN: Circular RNA splicing. Adv Exp Med Biol 1087: 41-52, 2018.

13. Du WW, Fang L, Yang W, Wu N, Awan FM, Yang Z and Yang BB: Induction of tumor apoptosis through a circular RNA enhancing Foxo3 activity. Cell Death Differ 24: 357-370, 2017.

14. Li Z, Huang C, Bao C, Chen L, Lin M, Wang X, Zhong G, Yu B, Hu W, Dai L, et al: Exon-intron circular RNAs regulate transcription in the nucleus. Nat Struct Mol Biol 22: 256-264, 2015.

15. Li F, Zhang L, Li W, Deng J, Zheng J, An M, Lu J and Zhou Y: Circular RNA ITCH has inhibitory effect on ESCC by suppressing the $\mathrm{Wnt} / \beta$-catenin pathway. Oncotarget 6 : 6001-6013, 2015.

16. Wang C, Tan S, Liu WR, Lei Q, Qiao W, Wu Y, Liu X, Cheng W, Wei YQ, Peng Y and Li W: RNA-Seq profiling of circular RNA in human lung adenocarcinoma and squamous cell carcinoma. Mol Cancer 18: 134, 2019.

17. Dube U, Del-Aguila JL, Li Z, Budde JP, Jiang S, Hsu S, Ibanez L, Fernandez MV, Farias F, Norton J, et al: An atlas of cortical circular RNA expression in Alzheimer disease brains demonstrates clinical and pathological associations. Nat Neurosci 22: 1903-1912, 2019.

18. Yang Y, Gao X, Zhang M, Yan S, Sun C, Xiao F, Huang N, Yang X, Zhao K, Zhou H, et al: Novel Role of FBXW7 circular RNA in repressing glioma tumorigenesis. J Natl Cancer Inst 110: 304-305, 2018.

19. Salzman J: Circular RNA expression: Its potential regulation and function. Trends Genet 32: 309-316, 2016.

20. Ameli-Mojarad M, Ameli-Mojarad M, Hadizadeh M, Young C, Babini H, Nazemalhosseini-Mojarad E and Bonab MA: The effective function of circular RNA in colorectal cancer. Cancer Cell Int 21: 496, 2021.

21. Tian T, Zhao Y, Zheng J, Jin S, Liu Z and Wang T: Circular RNA: A potential diagnostic, prognostic, and therapeutic biomarker for human triple-negative breast cancer. Mol Ther Nucleic Acids 26: 63-80, 2021.

22. Qu S, Song W, Yang X, Wang J, Zhang R, Zhang Z, Zhang H and Li H: Microarray expression profile of circular RNAs in human pancreatic ductal adenocarcinoma. Genom Data 5: 385-387, 2015.

23. Guo S, Xu X, Ouyang Y, Wang Y, Yang J, Yin L, Ge J and Wang H: Microarray expression profile analysis of circular RNAs in pancreatic cancer. Mol Med Rep 17: 7661-7671, 2018.

24. Li H, Hao X, Wang H, Liu Z, He Y, Pu M, Zhang H, Yu H, Duan J and Qu S: Circular RNA expression profile of pancreatic ductal adenocarcinoma revealed by microarray. Cell Physiol Biochem 40: 1334-1344, 2016.

25. Ritchie ME, Phipson B, Wu D, Hu Y, Law CW, Shi W and Smyth GK: Limma powers differential expression analyses for RNA-sequencing and microarray studies. Nucleic Acids Res 43: e47, 2015 .

26. van Roessel S, Kasumova GG, Verheij J, Najarian RM, Maggino L, de Pastena M, Malleo G, Marchegiani G, Salvia R, $\mathrm{Ng} \mathrm{SC}$, et al: International validation of the eighth edition of the American joint committee on cancer (AJCC) TNM staging system in patients with resected pancreatic cancer. JAMA Surg 153: e183617, 2018.

27. Livak KJ and Schmittgen TD: Analysis of relative gene expression data using real-time quantitative PCR and the 2(-Delta Delta C(T)) method. Methods 25: 402-408, 2001.

28. Crea F, Quagliata L, Michael A, Liu HH, Frumento P, Azad AA, Xue H, Pikor L, Watahiki A, Morant R, et al: Integrated analysis of the prostate cancer small-nucleolar transcriptome reveals SNORA55 as a driver of prostate cancer progression. Mol Oncol 10: 693-703, 2016.

29. Wang L, Sang J, Zhang Y, Gao L, Zhao D and Cao H: Circular RNA ITCH attenuates the progression of nasopharyngeal carcinoma by inducing PTEN upregulation via miR-214. J Gene Med: e3391, 2021 (Online ahead of print).

30. Zhu Z, Huang R and Huang B: Predicting functional circular RNA-based competitive endogenous RNA network in gastric carcinoma using novel bioinformatics analysis. Exp Biol Med (Maywood): 15353702211048757, 2021 (Epub ahead of print).

31. Chen Y, Li Z, Zhang M, Wang B, Ye J, Zhang Y, Tang D, Ma D, Jin W, Li X and Wang S: Circ-ASH2L promotes tumor progression by sponging miR-34a to regulate Notch1 in pancreatic ductal adenocarcinoma. J Exp Clin Cancer Res 38: 466, 2019. 
32. Guo X, Zhou Q, Su D, Luo Y, Fu Z, Huang L, Li Z, Jiang D, Kong Y, Li Z, et al: Circular RNA circBFAR promotes the progression of pancreatic ductal adenocarcinoma via the miR-34b-5p/MET/Akt axis. Mol Cancer 19: 83, 2020.

33. Xiao Y: Construction of a circRNA-miRNA-mRNA network to explore the pathogenesis and treatment of pancreatic ductal adenocarcinoma. J Cell Biochem 121: 394-406, 2020.

34. Li Q, Geng S, Yuan H, Li Y, Zhang S, Pu L, Ge J, Niu X, Li Y and Jiang H: Circular RNA expression profiles in extracellular vesicles from the plasma of patients with pancreatic ductal adenocarcinoma. FEBS Open Bio 9: 2052-2062, 2019.

35. Xu Y, Yao Y, Gao P and Cui Y: Upregulated circular RNA circ_0030235 predicts unfavorable prognosis in pancreatic ductal adenocarcinoma and facilitates cell progression by sponging miR-1253 and miR-1294. Biochem Biophys Res Commun 509: $138-142,2019$

36. Hao L, Rong W, Bai L, Cui H, Zhang S, Li Y, Chen D and Meng X: Upregulated circular RNA circ_0007534 indicates an unfavorable prognosis in pancreatic ductal adenocarcinoma and regulates cell proliferation, apoptosis, and invasion by sponging miR-625 and miR-892b. J Cell Biochem 120: 3780-3789, 2019.

37. Jiang Y, Wang T, Yan L and Qu L: A novel prognostic biomarker for pancreatic ductal adenocarcinoma: hsa_circ_0001649. Gene 675: 88-93, 2018.

38. Tang Q, Chen Z, Zhao L and Xu H: circular RNA hsa circ_0000515 acts as a miR-326 sponge to promote cervical cancer progression through up-regulation of ELK1. Aging (Albany NY) 11: 9982-9999, 2019.

39. Xia F, Chen Y, Jiang B, Bai N and Li X: Hsa_circ_0011385 accelerates the progression of thyroid cancer by targeting miR-361-3p. Cancer Cell Int 20: 49, 2020.
40. Ji Y, Sun Q, Zhang J and $\mathrm{Hu} \mathrm{H}$ : MiR-615 inhibits cell proliferation, migration and invasion by targeting EGFR in human glioblastoma. Biochem Biophys Res Commun 499: 719-726, 2018.

41. Guan X, Zong ZH, Liu Y, Chen S, Wang LL and Zhao Y: circPUM1 promotes tumorigenesis and progression of ovarian cancer by sponging miR-615-5p and miR-6753-5p. Mol Ther Nucleic Acids 18: 882-892, 2019.

42. Chen Y, Guo Y and Yan W: IncRNA RP5-916L7.2 correlates with advanced tumor stage, and promotes cells proliferation while inhibits cells apoptosis through targeting miR-328 and miR-939 in tongue squamous cell carcinoma. Clin Biochem 67: 24-32, 2019.

43. Chen A, Liu S, Lu X, Wei L and Chen Y: Inhibition of microRNA-939 suppresses the development of human non-small cell lung cancer via the upregulation of tissue inhibitor of metalloproteinases 2. Mol Med Rep 18: 4831-4838, 2018.

44. Rachinger N, Fischer S, Bohme I, Böhme I, Linck-Paulus L, Kuphal S, Kappelmann-Fenzl M and Bosserhoff AK: Loss of gene information: Discrepancies between RNA sequencing, cDNA microarray, and qRT-PCR. Int J Mol Sci 22: 9348, 2021.

45. Xu T, Wu J, Han P, Zhao Z and Song X: Circular RNA expression profiles and features in human tissues: A study using RNA-seq data. BMC Genomics 18 (Suppl 6): S680, 2017.

c) () $\ominus$ This work is licensed under a Creative Commons

c. Attribution-NonCommercial-NoDerivatives 4.0 International (CC BY-NC-ND 4.0) License. 